\title{
Adaptive Rewiring in Weighted Networks
}

\author{
Stefan Hellrigel, ${ }^{1,2}$, Nicholas Jarman ${ }^{2}$, Cees van Leeuwen ${ }^{2,1^{*}}$ \\ ${ }^{I}$ TU Kaiserslautern,Germany ${ }^{2} \mathrm{KU}$ Leuven, Belgium
}

*corresponding author:
cees.vanleeuwen@kuleuven.be

Tel.: +31.16 .326069$

Orcid: http://orcid.org/0000-0002-4441-2440

Acknowledgements:

SH, NJ and CvL are supported by Odysseus grant G.0003.12 from the Flemish Ministry of Science to $\mathrm{CvL}$. The authors wish to thank the anonymous reviewers for helpful suggestions. 


\begin{abstract}
Human connectome studies suggest that the brain has a modular small world network structure with rich-club effect. Such structure emerges spontaneously in simple model neural networks, (e.g. coupled maps), through adaptive rewiring according to the dynamic functional connectivity. The utility of adaptive rewiring has so far exclusively been demonstrated for unweighted networks; it is anything but guaranteed to work as well for weighted networks. We investigate adaptive rewiring in weighted networks, comparing various right-skewed, symmetrical, and left-skewed fixed weight distributions. We examine how network clustering, path length, modularity, and rich club coefficients develop for weakly, intermediate and strongly coupled networks. At low coupling strength, the weight distribution, as well as episodes of functional synchrony, have a significant effect on network evolution. With increased coupling strengths, all weighted networks robustly develop architectures similar to the unweighted ones. Adaptive rewiring appears relatively ineffective in networks with (biologically implausibly) extreme rightskewed weight distributions but performed most economically in biologically plausible log-normal distributions.
\end{abstract}

Keywords: complex adaptive systems; evolving networks; morphogenesis 


\section{Introduction}

Applications of graph theory have highlighted the ubiquity of small-world network structure (Watts \& Strogatz, 1998) in sociology (Gulati et al., 2012), biology (Lang et al., 2017), and, in particular, neuroscience (Varshney et al., 2011). Small-world structure has amply been demonstrated in brain connectivity networks (for a review, see Bassett \& Bullmore, 2017). Notably, in addition to small-world features (typically characterized by high clustering and low path-length), brain networks also exhibit the signatures of modular (Bertolero, Yeo, \& D’Esposito., 2015; Newman, 2004; 2006) and rich club organization (van den Heuvel, Sporns, Collin, et al., 2013).

Over the past fifteen years (starting with Gong \& van Leeuwen, 2003, 2004; van den Berg \& van Leeuwen, 2004) we have studied how these properties may become realized in the brain. Even in extremely simplified models, these properties emerge spontaneously from initially randomly organized networks. This occurs according to a principle, representing structural plasticity in the developing and adult brain (Butz, Wörgötter, \& van Ooyen., 2009). This principle has become known as adaptive rewiring (for a review see Papadopoulos, Kim, Kurths, \& Bassett, 2017).

Adaptive rewiring follows the Hebbian maxim: "what fires together, wires together". The way signals are communicated among network units (which may represent neurons or neuronal populations) involves dynamic coordination of their activity; in particular, synchronization (Fries, 2015). Adaptive rewiring establishes connections between synchronized units and removes connections between units that fail to synchronize their activity. Over time, the connections thus come to reflect the preferred communication pathways of the system (Rubinov, Sporns, van Leeuwen, \& Breakspear 2009). The resulting networks are typically modular small worlds (Rubinov et al., 2009; van den Berg \& van Leeuwen, 2004) and have one or more rich clubs (Jarman, Steur, Trengove, Tyukin, \& van Leeuwen, 2017), i.e. they are structurally similar to the brain.

Jarman et al. (2017) showed, moreover, that adaptive rewiring allows such network structures to emerge universally, i.e. from any form of dynamic activity diffusing on a network. Accordingly, these structures emerge in various artifical networks, including neural population oscillators with undirected connections (Gong \& van Leeuwen, 2004; Rubinov et al., 2009; van den Berg \& van Leeuwen, 2004) and spiking model neurons with directed connections (Kwok, Jurica, Raffone, \& van Leeuwen 2006). The universality observed in Jarman's study thus validates the use of simple models for complex biological systems like the brain. 
Adaptive rewiring, however, so far has exclusively been studied in un-weighted models, whereas brain network connections are typically represented as weighted networks. It is presently unknown, whether adaptive rewiring also leads to modular small worlds with rich clubs in weighted networks. Rewiring of weak (i.e. low-weighted) connections may have little effect on the structure; strong (highly-weighted) connections may show persistent synchronization that renders them resistent against rewiring. The relative prominence of strong and weak connections is expressed by the skewness of the weight distribution. We will investigate the effectivity of adaptive rewiring in left-skewed, symmetrical, and right-skewed weight distributions.

As several studies since Gong \& van Leeuwen (2003) have done, and licensed by the results of Jarman et al. (2017), we may represent without loss of generality the activity in network units by simple oscillators, i.e. coupled logistic maps (Kaneko, 1992). Logistic maps belong to the class of real-valued, non-linear dynamical equations that are updated in discrete time. Their dynamics may be considered a coarse approximation of oscillatory neural mass model actvity (Rubinov et al., 2009). Diffusive coupling between maps may represent interactions between neuronal units, for instance as realized through electrical synapses (gap junctions), ephaptic couplings, etc, as well as the dynamic synchronization between neural masses, as realized through macroscopic, intrapyramidal pathways. As we focus on interpyramidal connectivity, we use positive weights only. Diffusively coupled logistic maps give rise to a rich variety of collective activity patterns, including local and global spatiotemporal synchronization (Kaneko, 1992), similar to what is observed in spontaneous, largescale brain activity (Ito, Nikoleav, \& van Leeuwen, 2005; 2007).

Weighted, adjustable couplings have occasionally been used in globally coupled logistic maps (Ito \& Kaneko, 2002). Adaptive rewiring, however, operates in sparsely coupled networks. As in previous adaptive rewiring studies, the connectivity density (the total number of connections relative to the number of possible connections) and the number of nodes are kept constant for simplicity (but see Gong \& van Leeuwen, 2003 for adaptive rewiring in growing networks). We will compare weighted networks to previously studied unweighted networks. In this first application of adaptive rewiring to weighted networks, we assume the connection weight distribution to be fixed.

We consider a variety of weight distributions (Figure 1). Two of these are symmetrical: the uniform and Gaussian distributions. Connection weights have often been assumed to be Gaussian distributed; in the brain, however, right-skewed weight distributions, in particular lognormal ones, are understood to be more biologically plausible (Buzsáki \& Mizuseki, 2014; 
Roxin, Brunel, Hansel, et al., 2011; Song, Sjöström, Reigl, et al., 2005). These, therefore, are considered here, as well as power law weight distributions. The latter have no obvious biological plausiblity as they are extremely right-skewed but they are included in order to test the limits of adaptive rewiring effectivity under skewed distributions. For the sake of completeness, we also investigate left-skewed and extremely left-skewed distributions. We consider the inverses of the log-normal and power law distributions, respectively, obtained by mirroring these distributions in the vertical 0.5-plane (see Fig. 1).

Apart from the weights, network dynamics are controlled by a global coefficient: coupling strength. We compare the network evolution for a range of coupling strengths. To these networks, we apply unweighted and weighted network measures to test for small-world structure, modularity (Bertolero et al., 2015; Newman, 2004; 2006) and rich club effects (van den Heuvel et al., 2013). To show how the networks evolve as a function of the network dynamics, we compare their evolving structure with its fast and slow functional connectivity (Rubinov et al., 2009). The former involves the instantaneous patterns of synchrony; the latter the synchronous patterns over a longer time window in the network.

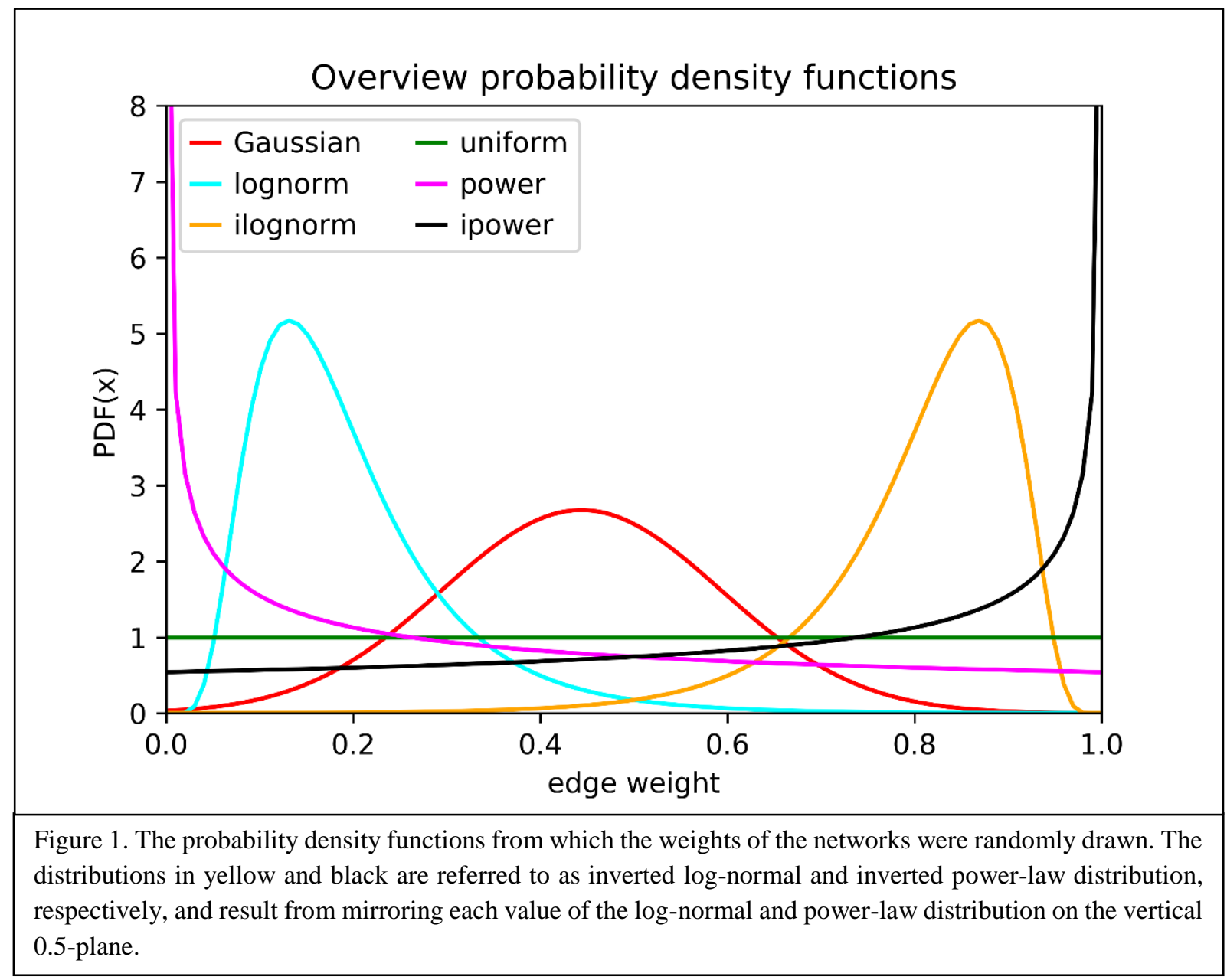




\section{Methods}

\section{Graph Theory}

In this section we motivate the use of graph theory as a means of analyzing our model networks. A graph is an ordered pair of sets $G=(V, E)$ where $v \in V$ are vertices and $E \subset\{(i, j) \mid i, j \in$ $V, i \neq j\}$ is the two element subset of $V$ called the edges (no self-loops are assumed). We assume the graphs to be undirected, $i . e .,(i, j)=(j, i)$. The total number of vertices and edges in the graph is $|V|=n$ and $|E|=e$, respectively. Two vertices $i, j \in V$ are called neighbours if $(i, j) \in E$. We define the set of neighbours $B_{i}$ of a given vertex $i$ as the set $B_{i}=\{i \in V \mid(i, j) \in E\}$. A weighted graph (or weighted network) is the ordered triple of sets $G=(V, E, W)$, where $V$ and $E$ are as defined, and $W=\left\{w^{i j} \in \mathbb{R}_{\geq 0} \mid(i, j) \in E\right\}$ is the set of edge weights.

\section{Dynamics on Graphs}

We call a vertex equipped with some expression of dynamics a node, and neighbouring nodes are said to be coupled. The dynamics of individual nodes is represented by iterations in discrete time of identical real-valued non-linear maps $f:[0,1] \rightarrow[0,1]$ of activity values $x_{i}(t)$, with $\mathrm{t}=0,1,2, \ldots$. Coupled node dynamics are described by the discrete iterative update process:

$$
x_{i}(t+1)=(1-\epsilon) f\left(x_{i}(t)\right)+\frac{\epsilon}{\sum_{j \in B_{i}} w^{i j}} \sum_{j \in B_{i}} w^{i j} f\left(x_{j}(t)\right)
$$

for nodes $i=1, \ldots, n$ and coupling strength $\varepsilon \in[0,1]$; specifically, we consider coupling strengths $\epsilon=0.3,0.4,0.5$. The logistic map $f\left(x_{i}(t)\right)=1-a x_{i}(t)^{2}$ denotes the state of node $i$ at time $t$ for a bifurcation parameter $a$. The parameter $a$ is kept fixed at $a=1.7$, for which the individual logistic map is chaotic. For such a parameter choice, the logistic map can be considered an extremely simplified model of neuronal population activity (Rubinov et al., 2009; van den Berg et al., 2012). Each node $i$ is influenced by its neighbours $j \in B_{i}$ proportional to the weight $w^{i j}$ of their edge $\left(w^{i j}>0\right)$. This proportion is normalized by the weighted degree $\sum_{j \in B_{i}} w^{i j}$ of node $i$ to maintain forward invariance of the dynamics, i.e., $x_{i}(0) \in[0,1]$ implies $x_{i}(t) \in[0,1], \forall t \geq 0$. 


\section{Edge Distribution}

The graphs considered consist of $|V|=300$ nodes, coupled by a total of $|E|=5200$ connections, resulting in a connectivity density of approximately 0.1159 . This connectivity density reflects the observed brain functional/structural connectivity (for reference see Gong \& van Leeuwen, 2004), and moreover, yields probability one for a random Erdös-Rényi network to be connected. In previous adaptive rewiring studies, such a connectivity density has proven sufficient for an unweighted network to successfully achieve small-world structure without becoming disconnected in the process (van den Berg, Gong, Breakspear, \& van Leeuwen 2012).

\section{Edge Weight Distribution}

For each run of the adaptive rewiring model, edge weights $w^{i j}$, for all $(i, j) \in E$, are randomly drawn from a specific probability distribution. As mentioned, distributions vary from extremely right-skewed (power law), via right-skewed (log normal), symmetrical (Gaussian and uniformly distributed) to left-skewed (inverted log-normal) and extremely left-skewed (inverted power-law) (See Fig. 1). For comparison, we include a model with unweighted edges, for which modular small-world networks have previously been shown to emerge (e.g. Gong \& van Leeuwen 2004).

To minimize the bias of the standard deviation and mean within individual distributions, distribution parameters are chosen such that their coefficients of variation $(\mathrm{CoV})$ are comparable (see Table 1). Minimizing differences in $\mathrm{CoV}$ trades off against how good the probability density functions are approximated. For instance, for a Gaussian distribution with $\operatorname{CoV}\left(X_{\text {Gaussian }}\right)=\frac{1}{3}$, the probability of randomly obtaing a negative weight is roughly 0.0027 . Since only positive weights are used, an average number of 14 weights per sample have to be re-assigned randomly. While a $\operatorname{CoV}\left(X_{\text {Gaussian }}\right)=0.5$ would more closely match the $\operatorname{CoVs}$ of the other distributions, the requirement that weights have positive values would have compromised the Gaussian distribution, since approximately 5\% of all the weights would have had to be re-assigned. 
Table 1: weight distributions used in the model simulations. CoV: coefficient of variation

\begin{tabular}{|c|c|c|c|c|}
\hline Distribution & Density Function $P(X)$ & $\begin{array}{c}\text { Parameter } \\
\text { values }\end{array}$ & $\mathrm{CoV}$ & $\sum$ weights \\
\hline Uniform & $\left\{\begin{array}{l}\frac{1}{b-a}, a \leq x \leq b \\
0, x<a \text { or } x>b\end{array}\right.$ & $\begin{array}{l}a=0, \\
b=1\end{array}$ & 0.58 & 2603.95 \\
\hline Gaussian & $\frac{1}{\sigma \sqrt{2 \pi}} e^{\frac{-(x-\mu)^{2}}{2 \sigma^{2}}}$ & $\begin{array}{l}\mu=3 \\
\sigma=1\end{array}$ & $\frac{1}{3}$ & 2348.40 \\
\hline Log-normal & $\left\{\begin{array}{c}\frac{1}{\sigma x \sqrt{2 \pi}} e^{-\frac{(\ln (x)-\mu)^{2}}{2 \sigma^{2}}}, x>0 \\
0, x \leq 0\end{array}\right.$ & $\begin{array}{c}\mu=0 \\
\sigma=0.5\end{array}$ & 0.53 & 891.58 \\
\hline Power-law & $\lambda x^{\lambda-1}, \lambda>0$ & $\lambda=0.5$ & 0.34 & 1738.49 \\
\hline \multicolumn{5}{|c|}{$\begin{array}{l}\text { Note: The weight distributions used in the model simulations. CoV: coefficient of variation. These } \\
\text { distributions were generated with the Python package SciPy (Jones et al., 2001). For a random } \\
\text { variable } X \text {, its methods are centered around parameters } s \text { (s=sigma; determines the shape of } \\
\text { the distribution), loc (offset in the } \mathrm{x} \text {-direction) and scale (representing } \mu \text { in the exponential). } \\
\text { We set } l o c=0 \text { and scale }=1 \text {, both for the power law and lognormal distributions., Because } \\
\text { model weights assume only positive values, the Gaussian distribution was shifted to the right } \\
\text { via loc }=3 \text { to secure that only a small number of selected values had to be redrawn, in order } \\
\text { to keep all values positive (see methods). We normalized all distributions to }(0,1] \text { to improve } \\
\text { readability and presentability. }\end{array}$} \\
\hline
\end{tabular}

\section{Adaptive Rewiring}

Rewiring the structure of the network takes place on a slower time scale than its dynamics. After each $T=20$ updates of the dynamics on the graph, a rewiring attempt is made according to Steps 3-5 of the procedure described below. Since rewiring attempts are not always successful, the ratio of rewiring and graph dynamics may vary.

- Step 0: Choose a probability density function and generate a weighted random graph $G=(V, E, W)$ of the Erdös-Rényi type, such that $|V|=n,|E|=0$, and $|W|=0$. Uniformly randomly select a pair of nodes $i, j$ from the set $\{i, j \in V \mid i \neq j,(i, j) \notin E\}$ and assign an undirected edge $(i, j)$ to $E$. Select randomly a weight $w^{i j}$ from a the chosen probability density function and assign it to $W$. Repeat until $|E|=5200$. 
- Step 1: Initialize each logistic map with a random value $x_{i}(0) \in[0,1]$ for $i=1, \ldots, n$.

- Step 2: Iterate each maps' dynamics according to (1) for transient time $T=20^{1}$.

- Step 3: Select uniformly at random node $i$, and define the synchrony errors $e_{i j}=$ $\left|x_{i}(T)-x_{j}(T)\right|, \forall j \in N, j \neq i$.

- Step 4: Identify nodes $k$ and $l$, such that $k=\operatorname{argmin}\left(\mathrm{e}_{\mathrm{ij}}\right), \forall \mathrm{j} \in \mathrm{V}$ and $l=$ $\operatorname{argmax}\left(e_{i j}\right), \forall j \in B_{i}$.

- Step 5: Attempt rewiring. If $(i, k) \notin E$, add $(i, k)$ with $w^{i k}=w^{i l}$ and remove $(i, l)$ from E.

- Step 6: Repeat steps 2-5 200000 times.

\section{Measures of Network Structure}

To analyze the effect of adaptive rewiring on the graph structure, we apply unweighted and weighted versions of the clustering coefficient and shortest path length, as well as unweighted and weighted measures for identifying hierarchical organization, i.e. modularity and rich-club coefficients.

The clustering coefficient $(C)$ is a measure of network segregation. A "triangle" centred around node $i$ is the complete subgraph of 3 nodes. The total number of triangles around a node $i$ is $T(i)$. A "potential triangle", centred on node $i$, is the subgraph $G_{i}^{\prime} \subset G$, such that $G_{i}^{\prime}=$ $\{i, j, k \in V \mid(i, j),(i, k) \in E,(k, j) \notin E\}$. The local clustering coefficient, $C_{i}=\frac{2 T(i)}{\mathrm{k}_{\mathrm{i}} k_{i-1}}$, is calculated for each node $i$ and represents the ratio of "number of triangles", $T(i)$, to "number of all possible triangles (triangles + potential triangles)", $\frac{\mathrm{k}_{\mathrm{i}} k_{i-1}}{2}$, with $k_{i}$ being the degree of node $i$. We report the global clustering coefficient for a whole network as the mean over all local clustering coefficients, such that $C=\frac{1}{n} \sum_{i \in V} C_{i}$.

The weighted clustering coefficient $(w C)$ is defined similarly. The local weighted clustering coefficient, $w C_{i}$ centred around node $i$, represents the relative influence of that triangle to the maximum weight in the network (Saramäki, Kivela, Onnela, et al., 2007).

\footnotetext{
${ }^{1}$ In previous studies much larger rewiring periods were used ( $\mathrm{T}=6000$ in Gong and van Leeuwen, 2004); $\mathrm{T}$ $=1000$ in Rubinov, Sporns et al., 2009). When optimizing the algorithm we established, however, that for T

$>10$, the rewiring period does not noticably affect the rewiring
} 
Therefore, $w C_{i}=\frac{\sum_{j, k \in B_{i}}\left(\hat{w}_{i j} \hat{w}_{i k} \hat{w}_{j k}\right)^{1 / 3}}{\mathrm{k}_{\mathrm{i}} k_{i-1}}$, where $\widehat{w}_{i j}=\frac{w_{i j}}{\max (w)}$. Consistent with our previous description, we report the global weighted clustering coefficient for the whole network as the mean over all local weighted clustering coefficients.

The average shortest path length $(L)$ is a classical measure of network integration. The shortest path length $d(i, j)$ between two nodes $i$ and $j$ in a binary network is the length of the shortest sequence of edges that connect $i$ to $j$. The average shortest path length over all pairwise nodes is $L=\sum_{i, j \in V} \frac{d(i, j)}{n(n-1)}$. A necessary condition to calculate the average shortest path length is that the network is connected (or fully connected in a directed network).

The weighted average shortest path length $(w L)$ measures network integration in weighted networks. This measure uses the inverse of the weights in the graph to calculate the weighted average shortest path length. A weighted path length between nodes $i$ and $j$ is the sum of inverted weights along a sequence of edges between the two nodes. We denote the weighted shortest path length between $i$ and $j$ as $d^{w}(i, j)$ as the minimum of all possible weighted path from $i$ to $j$. We report the weighted average shortest path length over all pairwise nodes, $w L=$ $\sum_{i, j \in V} \frac{d^{w}(i, j)}{n(n-1)}$.

The small-worldness coefficient $\Sigma=\frac{C / C_{\text {rand }}}{L / L_{\text {rand }}}$ is a compact means to report on clustering and path length at the same time. In cases where the measure might be misrepresentative (Rubinov \& Sporns, 2010) we report the average shortest path length and the clustering coefficient additionally. The weighted small-worldness coefficient is defined analogously as $w \Sigma=\frac{w C / w C_{\text {rand }}}{w L / w L_{\text {rand }}}$.

Modularity $(Q)$ (Newman, 2004; 2006; Saramäki et al., 2007) is a measure of network organization, representing the quality of a given subdivision of a network into non-overlapping communities. A community is typically a subset of nodes more densely intra-connected, while having fewer connections to nodes outside of the community. Modularity is formalised as $Q=$ $\frac{1}{2 e} \sum_{i, j \in N}\left[A_{i j}-\frac{k_{i} k_{j}}{2 e}\right] \delta\left(c_{j}, c_{i}\right)$ where $A_{i j}$ is the $n \times n$ weighted adjacency matrix, $k_{i}=\sum_{j} A_{i j}$ is the sum of the weights of the edges attached to vertex $i, c_{i}$ is the community to which vertex $i$ is assigned, the $\delta$-function $\delta\left(c_{i}, c_{j}\right)=\left\{\begin{array}{l}0, \text { if } c_{i} \neq c_{j} \\ 1, \text { if } c_{i}=c_{j}\end{array}\right.$. We use the Louvain community detection algorithm (Blondel, Guillaume, Lambiotte, et al., 2008), in which communities are constructed by the iterative merging of lower-level communities to maximise $\Delta Q$, the increase in modularity when merging two communities. 
The normalized rich-club coefficient $\left(\phi_{\text {norm }}(k)\right)$ characterizes a network's hierarchical organization. The value of this coefficient describes the extent to which nodes of (high) degree $k$ connect to other high degree nodes, forming a particularly influential subnetwork. Denote the rich-club coefficient $\phi(k)=\frac{2 e_{>k}}{n_{>k}\left(n_{>k}-1\right)}$, where $n_{>k}$ is the number of nodes having degree $\operatorname{deg}_{l}>k$ and $e_{>k}$ is the number of edges between such nodes with degree greater than $k$. Then, the normalized rich-club coefficient is the ratio $\phi_{\text {norm }}(k)=\frac{\phi(k)}{\phi_{\text {rand }}(k)}$, where $\phi_{\text {rand }}(k)$ is the expected rich-club coefficient for the randomly permuted graph that preserves the degree distribution at the same node degree level. To obtain reliable values for randomization, we construct 400 random networks (with the same degree distribution as the network under investigation), calculate their rich club coefficients $\phi_{i}(k), i=1, \ldots, 400$, and obtain $\phi_{\text {rand }}(k)=\sum_{i} \frac{\phi_{i}(k)}{400}$, the average rich-club coefficients at every degree level $k$. We denote $\phi, \phi_{\text {norm }}$ and $\phi_{\text {rand }}$ as the network-specific (normalized/randomized) sequence of rich club coefficients at consecutive node degrees. To test for statistical significance of rich club structures, we apply permutation testing (Bassett and Bullmore, 2009; van den Heuvel and Sporns, 2011), using the rich club sequence $\phi_{\text {rand }}$ as the distribution of the rich club coefficient under the null hypothesis (original sequence $=$ randomized sequence) to test it against $\phi$. We report statistical significance for $p<\alpha=.05$.

The normalized weighted rich-club coefficient $\left(\phi_{\text {norm }}^{w}(k)\right)$ is computed similarly. The value $\phi_{\text {norm }}^{w}(k)$ measures the extent to which connections between the high-degree nodes in the topological rich-club carry larger weights than expected by chance. We denote $\phi_{\text {norm }}^{w}(k)=$ $\frac{W_{>k}}{W_{>k}^{\text {rand }}}$, where $W_{>k}$ is the sum of all weights assigned to the edges of the rich club. Accordingly, $W_{>k}^{r a n d}$ is the sum of all weights assigned to the edges of the rich club of a randomised control network, so that $\phi_{\text {norm }}^{w}(k)>1$ indicates a normalized weighted rich club at degree level $k$. In this network, the topological structure is preserved but the weights are randomly assigned to arbitrary edges (Alstott, Panzarasa, Rubinov, et al., 2014). The normalization as well as the significance testing is achieved analogously to the topological rich club measure.

\section{Measures of Network Dynamics}

We describe the fast functional connectivity of the dynamics on the graph (Rubinov et al., 2009) in terms of matrix $S E$ of synchrony errors $s e_{i j}=\left|x_{i}-x_{j}\right|, j>i$ the Euclidean distance between the values $x$ of nodes $i$ and $j$. We then choose the $|E|=5200$ smallest entries of $S E$ and set all the other entries to 0 . With this procedure we construct synchrony error matrices, of which the entries represent the functional similarity between two nodes on a fast time scale. 
The number $|\mathrm{E}|=5200$ of non-zero entries is chosen to match the connectivity density of the underlying structural network.

To represent slow functional connectivity, we performed 100 iterative updates of the dynamics on all nodes on a fixed network structure. We then constructed a correlation matrix $\mathrm{CM}$ with entries $c m_{i j}=\rho\left(X_{i}, X_{j}\right), j>i$, the Pearson correlation coefficients for a string of 100 consecutive updates of dynamics $x$ of nodes $i$ and $j$. We choose the $|\mathrm{E}|=5200$ most strongly correlated entries and set the other values to 0 , to construct slow functional connectivity matrices matching the connectivity density of the structural network.

We investigate the difference between functional connectivity matrices by utilizing the Frobenius matrix norm (Golub \& Van Loan, 1996, p.55). The Frobenius norm of an (mxn)real-valued matrix $A$ is defined as $\left\|A_{F}\right\|=\sqrt{\sum_{i=1}^{m} \sum_{j=1}^{n} a_{i j}^{2}}$, the square root of the sum of the squared matrix elements $a_{i j}$. For two functional connectivity matrices $F C M_{t}$ and $F C M_{t+k}$ at time $t$ and $t+k$, the Frobenius norm $\left\|F C M_{t}-F C M_{t+k}\right\|_{F}$ is an inverse measure of their similarity.

\section{Software}

We use the Python package NetworkX (Hagberg, Swart, \& Chult, 2008) to compute (weighted and unweighted) clustering coefficient, (weighted and unweighted) path length, and topological (i.e. unweighted) rich-club coefficient. For the analysis of the modular structure of the networks, we use the Python package community (Aynaud, 2017). For the analysis of the weighted rich-clubs we apply the normalization method as presented by Alstott, et al., (2014).

\section{Data Collection}

The adaptive rewiring model was ran in all combinations of three coupling strength values and six edge weight distributions. Each run was repeated five times, measures observed are averages from five runs; vertical bars represent standard errors. We obtained clustering coefficients $w C$ (weighted) and $C$ (unweighted), path lengths $w L$ (weighted) and $L$ (unweighted), and small-worldness $w \Sigma$ (weighted) and $\Sigma$ (unweighted) as well as the mean average of the weighted and unweighted modularity $\mathrm{w} Q$, Q respectively. We collected unaveraged results of characteristic single runs for the topological (unweighted) and weighted rich-club coefficients $\phi_{\text {norm }}$ and $\phi_{\text {norm }}^{w}$, respectively. For further single runs, we plotted structural and functional connectivity matrices and compared the evolution of network structure with that of the fast functional connectivity in terms of the Frobenius norm $\left\|F C M_{t}-F C M_{t+1}\right\|_{F}$. 


\section{Results}

\section{Clustering}

Figure 2 shows the evolution of the weighted and unweighted clustering coefficients for all weight distributions and coupling strengths. Both coefficients are normalized by their values of the initial random network $\left(w C_{0} / C_{0}\right)$. For the weakest coupling strength, $\varepsilon=0.3$, the Gaussian and inverse log-normal networks develop a highly clustered organization, comparable to that of the unweighted (i.e. binary) networks. The inverse power-law, log-normal, and
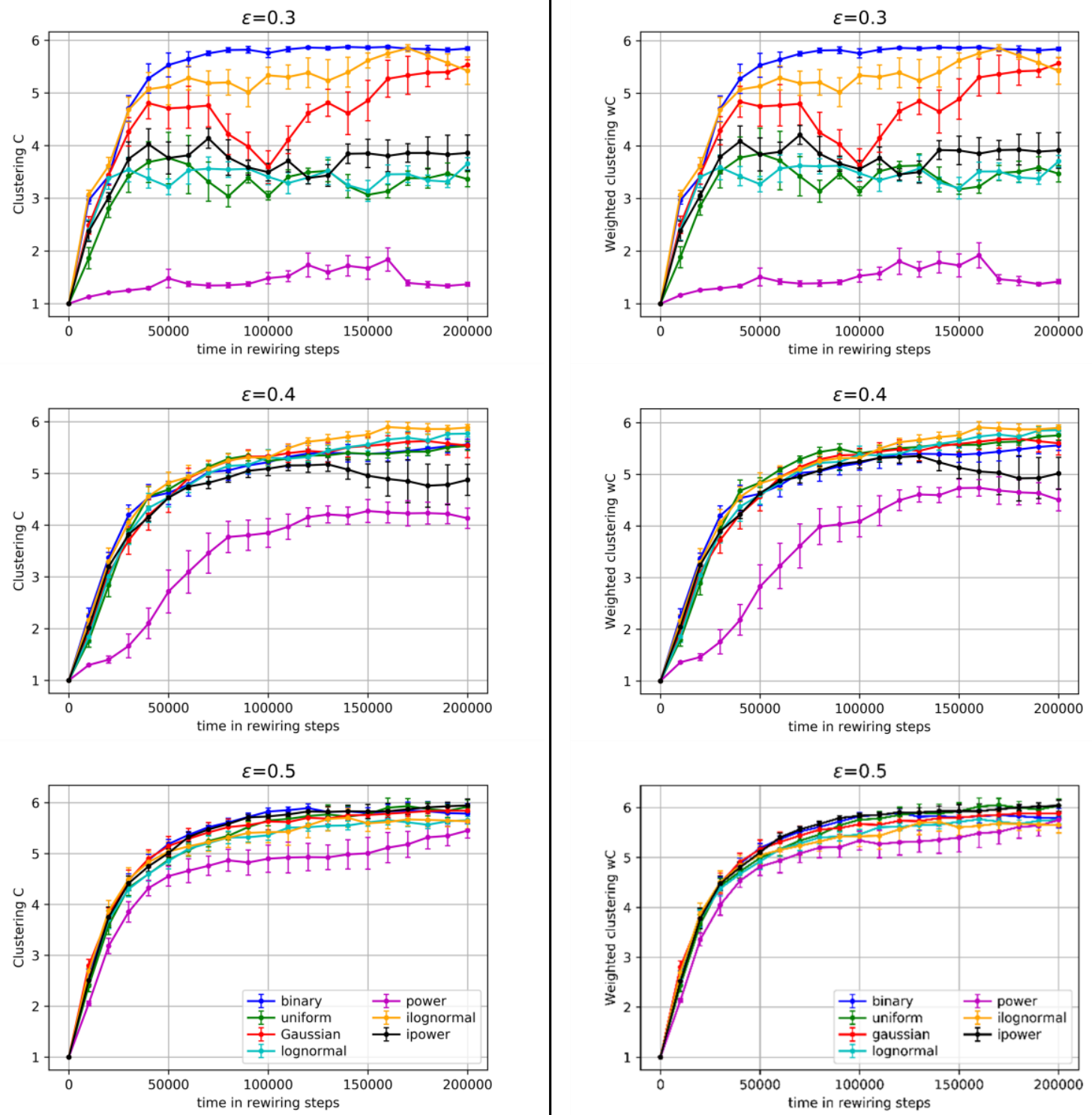

Figure 2. Development of the clustering coefficient (left column) and weighted clustering coefficient (right column) over time for each distribution at all three levels of coupling strength $\varepsilon$ (rows). We present normalized coefficients $w C / w C_{0}$ (right column) and $C / C_{0}$ to facilitate th comparison of network evolution under different weight distributions. 
uniform distribution reach intermediate clustering values. In the power-law distribution, clustering fails to increase at this coupling strength.

In unweighted networks, van den Berg et al. (2012) showed that the evolution of clustering becomes critically unstable and eventually unsuccessful, when connectivity becomes increasingly more sparse. One possibility, therefore, is that low-weights are negligible, thereby increasing the effective sparseness of the weighted networks, as far as rewiring is concerned. This would be particularly damaging to the network with the largest proportion of low-weighted connections, i.e. the power law network.

Close inspection suggested that, in general, initial increases in clustering in power-law networks are accompanied by stable episodes of widespread synchrony, which can last up to
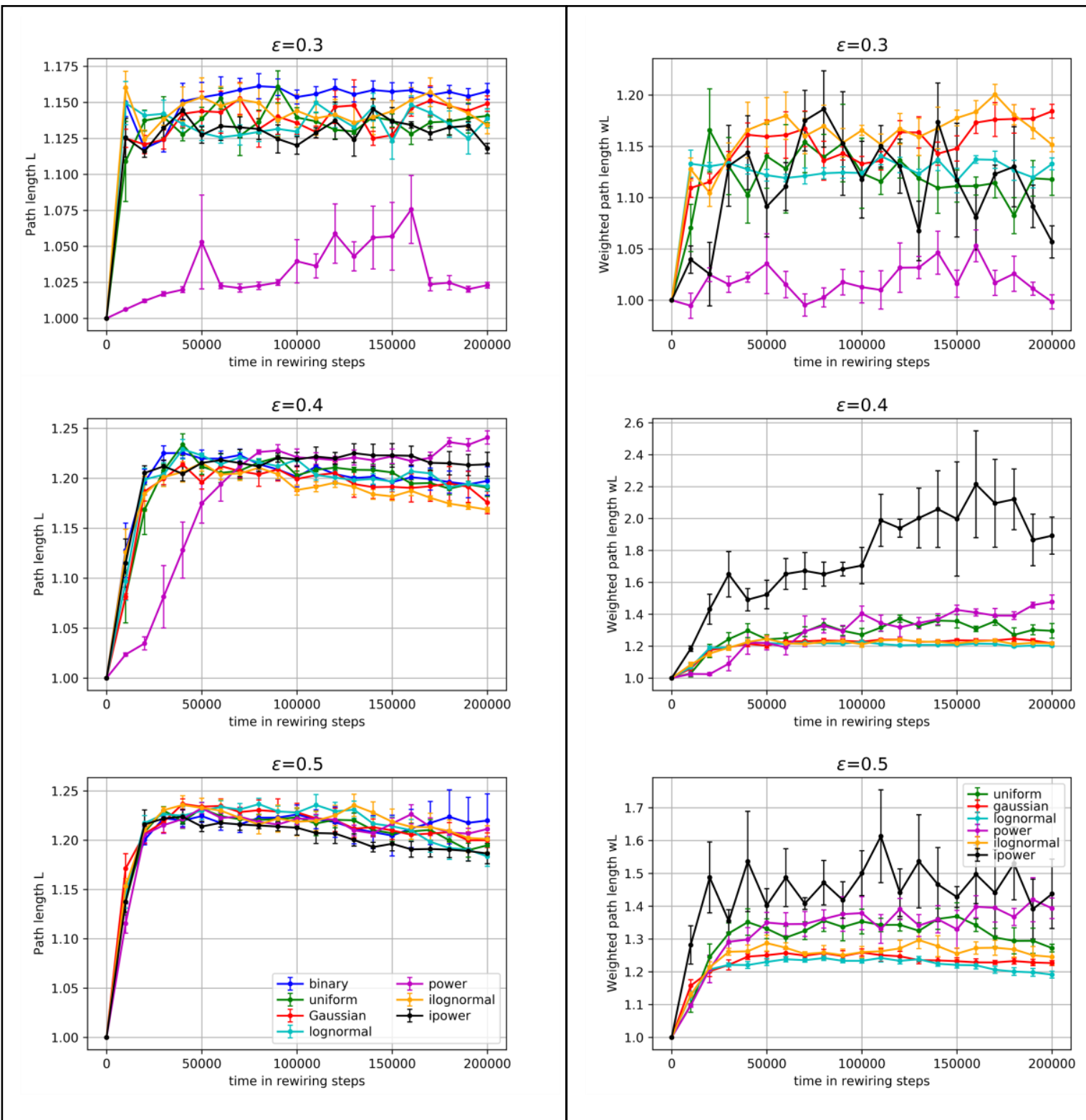

Figure 3. Development of the average shortest path length (left column) and the weighted average shortest path length (right column) of each distribution. As with the weighted clustering coefficient, we normalize the values $w L_{t}$ and $L_{t}$ at time $t$ by $w L_{0}$ and $L_{0}$, respectively, of the initial random networks. 
80 rewiring attempts (successful or failed). With widespread synchrony, weak and strong connections have roughly equal probabilities of being rewired. Power-law distributed networks seem to depend for their evolution more strongly than other distributions on such episodes. We will study this phenomenon more extensively in the last part of the results section, devoted to the relation between structural and functional connectivity.

For $\varepsilon=0.4$, networks with inverse power-law, log-normal, and power-law distributed weights now achieve clustering values similar to the unweighted (binary) networks. These networks reach their evolved states later than with coupling strength $\varepsilon=0.3$. For $\varepsilon=0.5$, the evolution of the clustering coefficient is more stable than in both previous conditions; all weight distributions are able to establish and maintain relative clustering coefficient values above 5.

\section{Path Length}

Figure 3 shows that for the weakest coupling $(\varepsilon=0.3)$, path length $L$ evolves similarly across weight distributions, except for power-law networks. Their path length remains essentially at the level of random networks, but with some abrupt, transient lengthening. Stronger coupling $(\varepsilon=0.4 ; 0.5)$ leads to path length increasingly evolving rather uniformly. The same could be said about weighted path length $w L$, except for the power-law weightdistributed networks. At $\varepsilon=0.4$, some of these networks show (from early on) very sharp
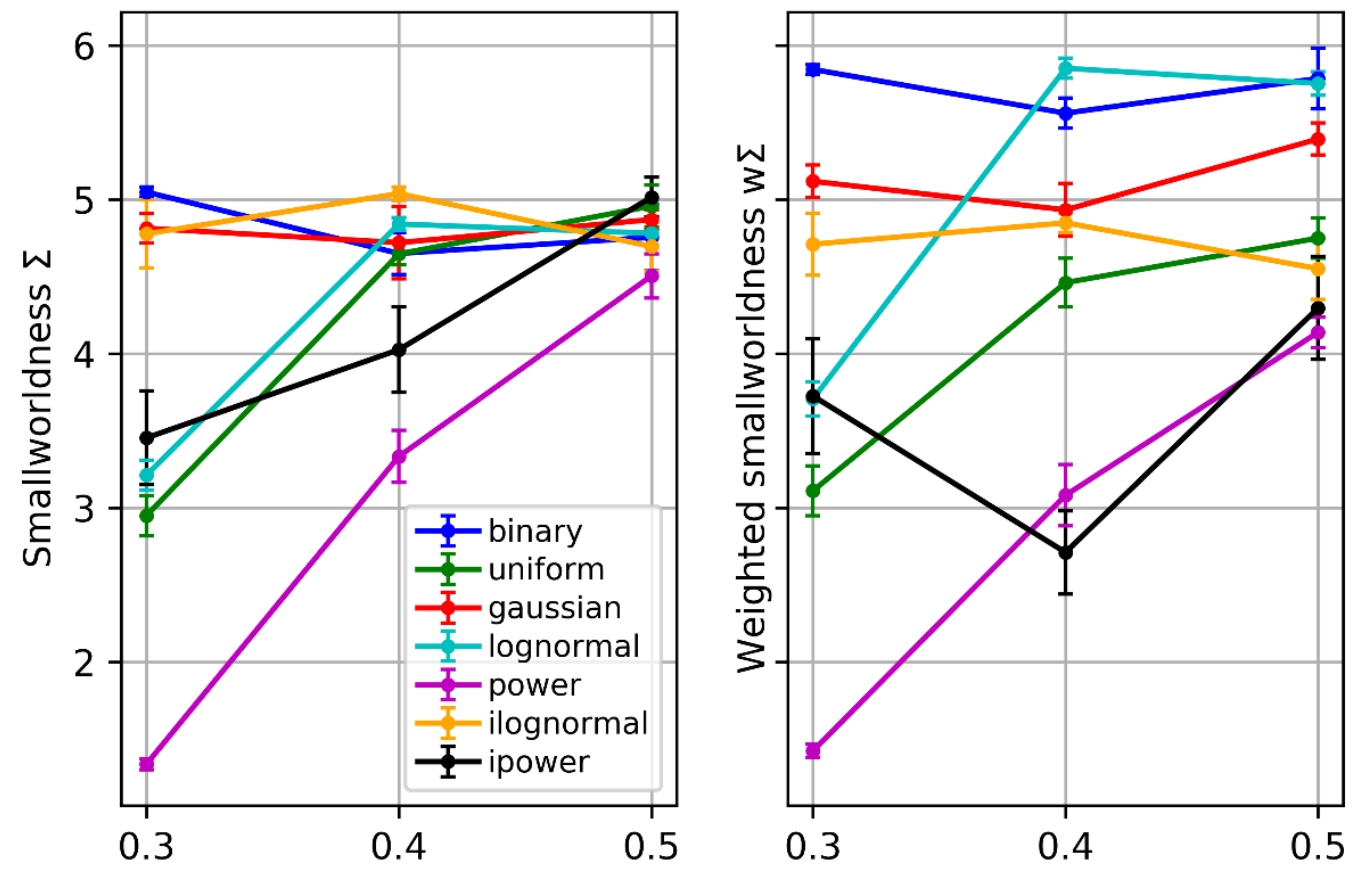

Coupling strength $\varepsilon$

Figure 4. Unweighted (left) and weighted (right) small-worldness of the final networks for all three levels of coupling strength. The reported values are averaged over five samples. 
increases in weighted path length. Their strongest connections tend to cluster together at the expense of integration in the remaining network. The same applies, to a lesser extent, for $\varepsilon=0.5$.

\section{Small-worldness}

The small-worldness coefficicients, which combine the clustering and path-length measures, concisely reflect our main observations: considerable variation across the weight distributions for weak coupling $\varepsilon=0.3$ with, in particular, the power-law falling behind the others. With increased coupling strengths, the variance between weight distributions decreases, especially for the unweighted measure. Unweighted (binary) networks invariably evolve to small-worlds independently of coupling strength. Overall, we may conclude that the formation of small-world networks is only moderately affected by the presence of different weights.
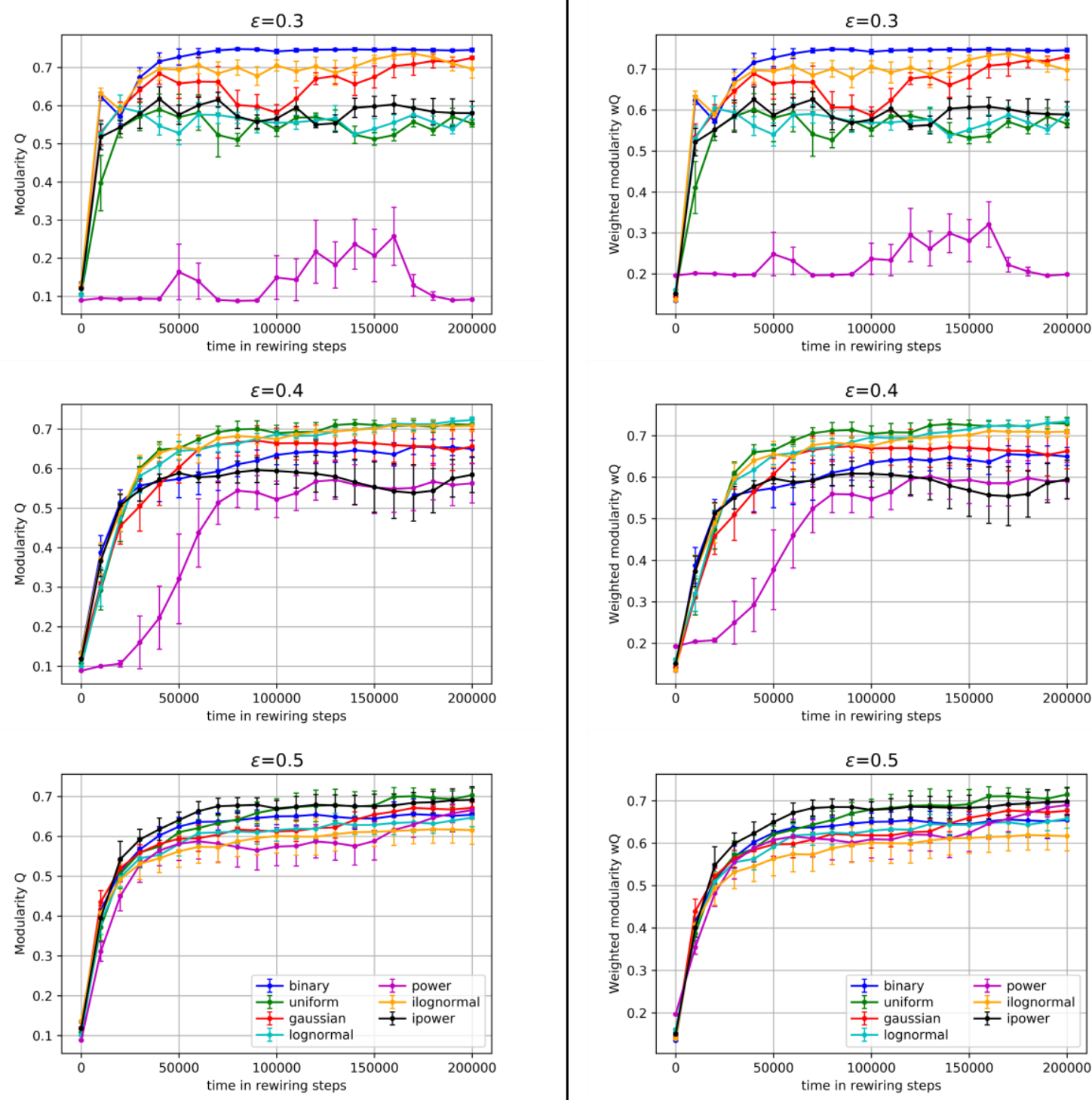

Figure 5. Evolution of the modularity index $Q$ (left) and the weighted modularity index $w Q$ (right) for all weight distributions as a function of rewiring attempts. Because the modularity landscape shows neardegeneracy (Good de Montjoye \& Clauset, 2010), each modularity value is the mean over 30 calculations of modularity for each rewiring attempt and run. Vertical bars indicate standard errors. 


\section{Modularity}

The pattern of results for modularity is consistent with that of clustering, path length, and small world structure. As Figure 5 shows, for the weak coupling $(\varepsilon=0.3)$, modularity evolution depends strongly on the weight distribution, with power-law networks failing entirely to develop into modular networks. With coupling strength $\varepsilon=0.4$, all weight distributions develop modular structures to various extents. For this coupling strength, modularity develops more slowly than for the weakest coupling, apparently as a result of an increased failed rewirings rate. For the highest coupling strength, $\varepsilon=0.5$, modularity develops rather uniformly across different weight distributions.

clustering and path-length

\section{Rich Club}

We investigated 105 different networks (7 distributions x 3 levels of coupling strength x 5 individual runs); an in depth analysis of every network is beyond the scope of this article. For illustration, Figure 6 presents rich-club coefficients of individual networks as a function of node degree (i.e. its number of connections). The leftmost panels show the normalized topological (i.e. unweighted) rich-club coefficients.

We find significant topological rich club structure mostly towards the higher end of the degree sequence, for example in a Gaussian network (from $k=19$ till the end; $p=0.00012$, permutation test) or in a lognormal network (from $\mathrm{k}=18$ till the end; $\mathrm{p}=0.0001$, permutation test), even at coupling strength $\varepsilon=0.3$. Power-law networks at this coupling strength are inconsistent. Some form significant rich clubs with a lower peak compared to the other networks (from $\mathrm{k}=13$ till the end; $\mathrm{p}=0.01683$, permutation test), while others do not evolve any significant rich clubs (from $\mathrm{k}=18$ till $\mathrm{k}=61 ; \mathrm{p}=0.16656$, ns., permutation test).

For the higher coupling strengths, results become unambiguous. All networks develop significant topological rich-club structure. These tend to start earlier in the degree sequence than for weak couplings. In addition, some networks develop significantly longer degree sequences than others (see Fig. 6), which, however, does not affect the significance of the statistics (power-law network at $\varepsilon=0.4$ : from $k=5$ till the end $(k=97), p<0.0001$, permutation test).

In sum, topological rich-clubs consistently arise for most weight distributions, except for power law distributed networks at the lowest coupling strength. This result is consistent with the special status of these networks, as observed through other measures. 
The rightmost panels of Figure 6 show normalized weighted rich club coefficients as a function of node degree for representative samples. Unlike the topographical rich clubs, weighted rich-clubs are unsystematic in their appearance across weight distributions and coupling strengths.

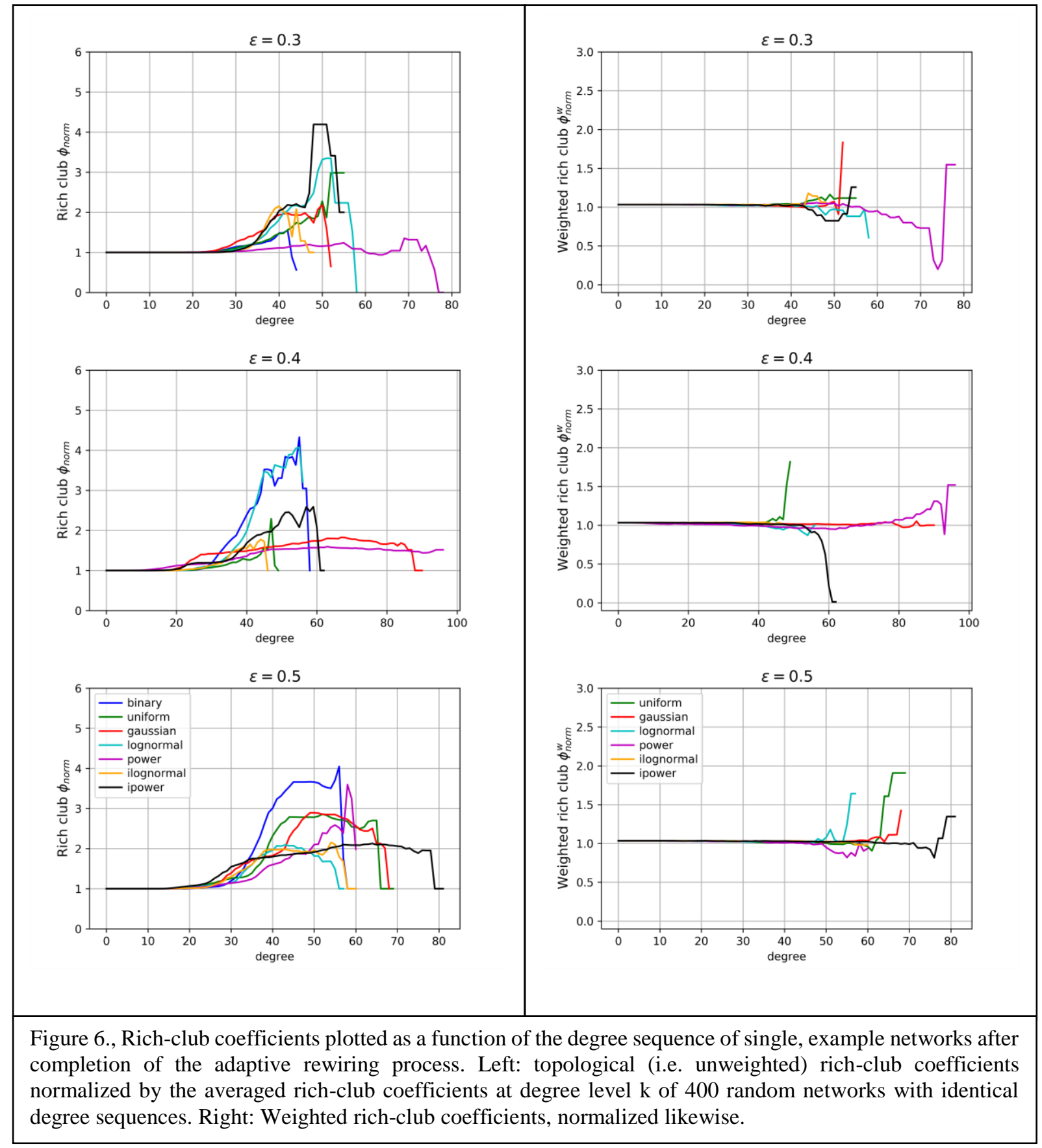

\section{Structural and Functional Connectivity}

For unweighted networks, Rubinov et al. (2009) considered the evolution of the adaptively rewiring structure in relation to the fluctuations in, respectively, the fast and slow 


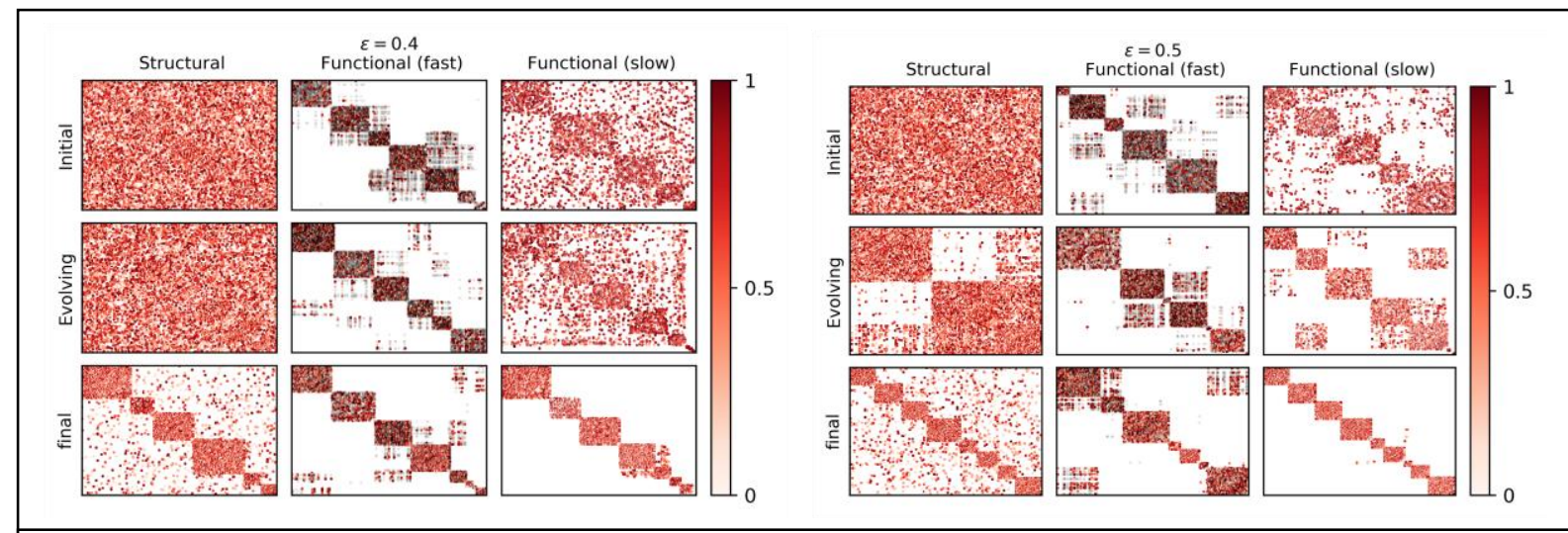

Figure 7. Example power law networks with coupling strength $\varepsilon=0.4$ (left panel) and $\varepsilon=0.5$ (right panel). Structural (left columns), fast functional (mid columns) and slow functional (right columns) connectivity networks during three stages of the adaptive rewiring process: initial (top row), evolving (mid row) and final (bottom row). To obtain the leftmost, mid and right column, weight values, thresholded synchrony errors, and correlations, respectively, were normalized and aligned, such that 0 indicates minimal, 1 maximal strength. Units were rearranged to highlight the formation of modules throughout the rewiring process., The colorcoding of an edge represents the percentile of its weight in its distribution. The functional connectivity networks are not bound to the structural network, such that functional connections may occur that are not represented by a structural edge. These are represented by black dots.

functional connectivity (i.e. the instantaneous and time-windowed patterns of synchrony in the network). They observed convergence over time between, on the one hand, the modular structure of the network and, on the other, the preferred intramodular synchronization patterns in the slow functional connectivity. At the same time, the structural networks preserve pathways for intermodular communication sufficient to retain the integrity of the small world network.

Corresponding patterns of intermodular synchrony are not typically observed in the slow functional connectivity. Because the fast functional connectivity patterns continue to fluctuate independently of the structural modules, cross-modular synchronization patterns are established in the fast functional connectivity. These are sufficient for adaptively creating and maintaining the structural inter-modular connectivity patterns. Rubinov et al. (2009) summarily described these complementary effects of network synchronization on network evolution as a symbiosis of structural and functional connectivity.

We investigated whether such a symbiosis occurs also for weighted networks. Of particular interest is the power law distributed network, of which the robustness of the evolution depends on coupling strength; modularity fails to appear for $\varepsilon=0.3$, is weak for $\varepsilon=0.4$ and robust for $\varepsilon=0.5$. Figure 7 depicts three stages in the evolution of power law networks with coupling strengths $\varepsilon=0.4$ and $\varepsilon=0.5$, respectively.

Leftmost columns show the evoluton of the graph structure. Note that the total number of connections remains constant throughout the evolution. The apparent greater connectivity density in the initial conditions is merely a by-product of pixel size. For $\varepsilon=0.4$, the symbiosis effect is only partially expressed. Several structural modules match the patterns of synchrony 
in the slow functional connectivity, but some modules show only partial synchronization. The fast functional connectivity patterns are, like in Rubinov et al. (2009), independent of the modular structure. As a result, the network structure retains a relatively large number of intermodular connections. For $\varepsilon=0.5$, the modular structure converges with the patterns of synchrony in the slow functional connectivity. The intermodular connectivity structure becomes more sparse. Still, their number is sufficient to sustain an integrated small world structure. We conclude that weighted networks realize their structure through a symbiosis of functional and structural connectivity, similarly to the unweighted ones in Rubinov et al. (2009). This, however, only as long as the coupling strenth is sufficient to overcome the adverse effects of the weighting, which are particularly prominent in the power law network.

Interestingly, the intermodular connections are, as demonstrated here in particular for the highly skewed power law distribution, mostly from the lower part of the weight distribution. Given that low weights are unlikely to support stable patterns of synchrony, their role in the slow functional connectivity is limited. For this reason, they are relatively unlikely to become part of the intra-modular connectivity structure. The prominence of weak connections in the distribution therefore explains why power law networks are relatively diffult to organize into modular small-worlds. On the other hand, increasing the coupling strength raises the likelihood of synchrony for all connections, including the weaker ones, allowing them to become absorbed into modules.

To further illustrate the role of the fast functional connectivity in network evolution, Figure 8 shows network evolution aligned with fluctuations in synchrony for specific runs of two distribution types: the power law (top panels) and the inverse lognormal (bottom panels). To characterize these fluctuations, we use the Frobenius norm $\left\|F C M_{t}-F C M_{t+1}\right\|_{F}$ of the difference between the fast functional connectivity matrices (FCM) at subsequent times. The higher the norm, the stronger the fluctuations in the fast functional connectivity.

Moment to moment synchrony in our networks typically show some brief periods in which the fast functional connectivity is (relatively) stable. Such periods give a boost to the formation of small world structure. This process is illustrated in the top-left panel of Figure 8. The weakly connected power law network, which normally fails to develop substantial degrees of clustering, shows a temporary increase in the clustering coefficient after periods in which the fast functional connectivity was stable. But under the normally high fluctuations in synchrony, this structuure cannot be sustained. This phenomenon resembles the failure of clustering with unweighted networks with insufficient connectivity desnsity in van den Berg et al. (2012). The power law network has a large number of low-weighted connections, which may amount to 
underconnectivity if also the coupling is weak. The top-right panel shows that with higher coupling strength, the growth of clustering in the power law network is unimpeded by these fluctuations.

For the weakly coupled inverse lognormal network (bottom-left panel), the effect of stability in the fast functional connectivity is the opposite: it initiates a (temporary) decline in clustering. Yet, in this network the growth in clustering is robust under normal fluctuations, and capable of maintaining a high degree of clustering, as the lower-right panel shows).

The opposite effect of stable periods in the functional connectivity may be associated with the opposite character of the distributions. Low weights predominate in the power law networks; high weights in the inverse power law networks. The stability of the fast functional connectivity may faciltate a temporary clustering of the low-weight connections in the network, which is subsequently destabilized as the fluctuations in the fast connectivity resume.

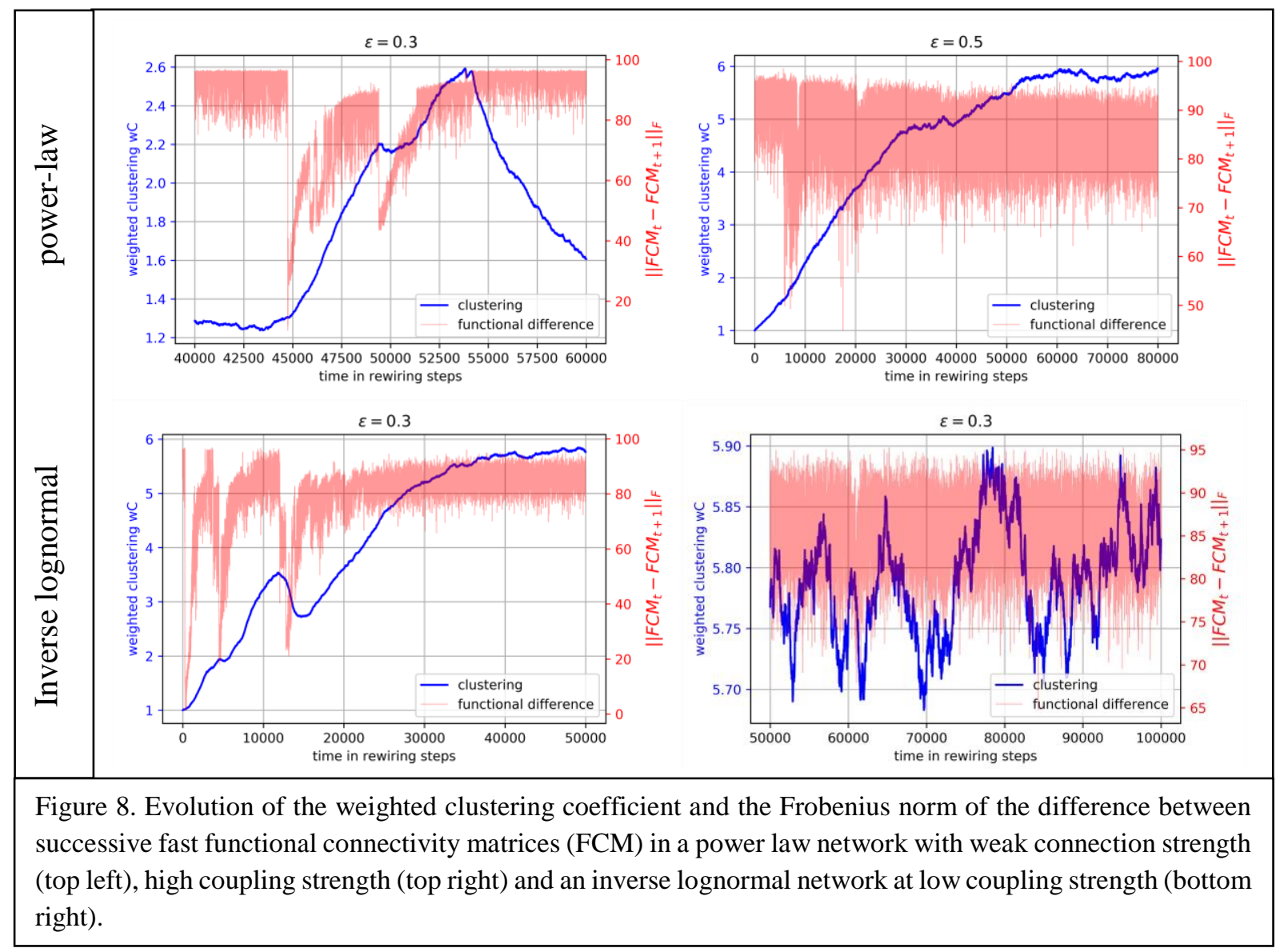

\section{Discussion}

Adaptive rewiring offers an elegant way for representing in neural network models the evolution of network connectivity structure of the human brain. By showing how adaptive rewiring operates in weighted networks, we have made a step towards greater neural realism of 
such models. Different weight distributions were compared, ranging from extremely leftskewed, over symmetrical, to extremely right-skewed ones. Compared to unweighted, binary network connections, weighted connections only mildly affect the evolution to small worlds. For most part, weighted networks evolved, like binary ones, to modular small-worlds with rich club effects.

Amongst the weight distributions we considered, the power-law networks performed worst. These were included in our study in order to gain an understanding of how extreme skewedness may affect network evolution, but possess no biological relevance. Networks with log-normal distributed weights are generally considered the ones most neurally plausible (Buzsáki \& Mizuseki, 2014; Roxin et al., 2011; Song et al., 2005). These networks perfomed only average in adaptive rewiring, compared to the other weight distributions. Previous work has shown numerous advantages of log-normal weight distributions, ranging from sparsity in spontaneous firing rates (Ikegaya, Sasaki, Ishikawa, et al. 2013) to coding for visual information (Cossell, Iacaruso, Muir et al. 2015). But as it stands, the current results seem to suggests no specific advantage of lognormal weight distributions over uniform or Gaussian weights, regarding adaptive rewiring.

On the other hand, it could be argued the log-normal weight distribution performs just as well as the other ones, but in a more efficient way. Its average weight is the lowest of all the distributions considered. Weights may represent a range of different neuronal properties, such as average firing rate (Ikegaya, et al. 2013), persistency (Ortiz, Górriz, \& Ramírez, 2017) or distance (Muldoon, Bridgeford, \& Bassett, 2016); in all these cases, however, we may interpret the sum of weights as a measure of biological cost. This makes the log-normal distribution the most cost efficient weight distribution for networks that succesfully perform adaptive rewiring. Coupling strength turned out to be the moderator variable for the effect of the weight distribution on the emergence of small-world networks. While the evolution is robust for binary networks with weak couplings, it is unstable to various degrees for weighted networks, and absent for networks with power law distributed weights. For intermediate and strongly coupled networks, the behavior of the weighted networks increasingly becomes similar to that of binary networks for all weight distributions, including the power law distribution.

The dependency on coupling strength could be explained as follows: The more strongly coupled a networks units are, the more likely they are to reach synchrony. The spreading of stable synchrony helps overcome the negative effects of the weight distribution, as we have seen in the extreme case of networks with power law distributed weights. These suffer from the resistance to rewiring of the few strongly weighted connections and the relative ineffectivity of 
the remaining ones. The latter has the same effect as reducing the network connectivity density, which, below a critical threshold, can no longer establish clustering (van den Berg et al., 2012). As shown for this network in Figure 7, the system fails to trigger the symbiosis of the long term functional connectivity and the network structure that maintains the network clustering (Rubinov et al., 2009).

As ilustrated in the leftmost panels of Figure 8, periods in which stable synchronization spreads globally occur spontaneously in our networks of coupled oscillators. Nodes connected with high weights generally have a high probability of being synchronized, which prevents their connection from being rewired. Periods in which the synchrony spread reduce this protection, enabling such weighted connections to be rewired. This gives an initial boost to the clustering which, for most of our weight distributions, is sufficient to trigger the symbiosis that allows clusterig to be increased. For weakly coupled power law networks, this boost, however, is insufficient. The few extremely high weights are concentrated within a small population of nodes, making them particularly resistant against rewiring.

Like the activity in our models, spontaneous brain activity is characterized by intermittent episodes of globally correlated activity, consisting of traveling and standing waves (Ito et al., 2005; 2007; Gong et al., 2007). We propose that these episodes help in reorganizing network connectivity, in particular of some dominant connections. Traveling waves are also observed in evoked (Alexander, Jurica, Trengove, et al.2013) and eye-movement related activity (Giannini, Alexander, Nikolaev, et al., 2018), where they establish a rapid, taskdependent reorganization of the functional connectivity (Seidkhani, Nikolaev, Meghanathan, 2017).

By showing that adaptive rewiring is effective in weighted networks, we have extended its scope as a basic principle of morphogenesis in structural and functional networks. Whereas the present study prioritizes generality over realism, incorporating weights has brought this principle an important step closer to application to biologically realistic network models. 


\section{References}

Achard S, Delon-Martin C, Vértes PE, Renard F, Schenck M, Schneider F, Heinrich C, Kremer S, Bullmore ET (2012) Hubs of brain functional networks are radically reorganized in comatose patients Proc Nat Acad Sc USA 109:20608-20613

Alexander DM, Jurica P, Trengove C, Nikolaev,AR, Gepshtein S, Zvyagyntsev,M, Mathiak K, Schulze-Bonhage A, Rüscher J, Bal, T, \& van Leeuwen C (2013) Traveling waves and trial averaging; the nature of single-trial and averaged brain responses in large-scale cortical signals NeuroImage 73: 95-112.

Alstott J, Panzarasa P, Rubinov M, Bullmore ET, Vertes, PE (2014) A unifying framework for measuring weighted rich clubs Sci Rep 4:7258

Aynaud T (2017) Python-louvain 0.8 https://pypi.python.org/pypi/python-louvain/0.8 version uploaded 06-03-2017

Bassett DS, Bullmore ET (2009) Human brain networks in health and disease. Curr Opin Neurol 22: 340-347

Bassett DS, Bullmore ET (2017) Small-World Brain Networks Revisited. The Neuroscientist 23:499-516. doi:10.1177/1073858416667720.

Bertolero MA, Yeo BTT, D'Esposito M (2015) The modular and integrative functional architecture of the human brain. Proc Nat Acad Sc USA 112: E6798-E67807,

Blondel VD, Guillaume J-L, Lambiotte R, Lefebvre E (2008) Fast unfolding of communities in large networks J Stat Mech P10008

Bolaños M, Bernat EM, He B, Aviyente S (2013) A weighted small world network measure for assessing functional connectivity $J$ Neurosc Meth, 212:133-142

Brandes U (2008) On Variants of Shortest-Path Betweenness Centrality and their Generic Computation. Social Networks 30(2):136-145

Bullmore E, Sporns O (2009) Complex brain networks: graph theoretical analysis of structural and functional systems. Nat Rev Neurosc 10:186-198

Butz M, Wörgötter F, van Ooyen A. (2009) Activity-dependent structural plasticity Brain Res $\operatorname{Rev}$ 60: 287-305

Buzsáki G, Mizuseki K (2014) The log-dynamic brain: how skewed distributions affect network operations. Nat Rev Neurosc 15:264-278

Cossell L, Iacaruso MF, Muir DR, Houlton R, Sader EN, Ko H, Hofer SB, Mrsic-Flogel TD (2015) Functional organization of excitatory synaptic strength in primary visual cortex. Nature 518:399-403

Fries P (2015) Rhythms for cognition: communication through coherence. Neuron 88: 220 235

Giannini M, Alexander DM, Nikolaev AR, van Leeuwen, C (2018) Large-scale traveling waves in EEG activity following eye movement Brain Topogr https://doi.org/10.1007/s10548-018-0622-2.

Golub GH, Van Loan CF (1996) Matrix Computations, 3rd ed. Baltimore, MD: Johns Hopkins.

Good BH, Montjoye Y-A de, Clauset A (2010) Performance of modularity maximization in practical contexts. Phys Rev E 81:046106

Gong P, Nikolaev AR, van Leeuwen C (2007) Dynamics of collective phase synchronization in human electrocortical activity. Phys Rev E 76:011904. 
Gong P, van Leeuwen C (2003) Emergence of scale-free network with chaotic units Phys A Stat Mech Appl 321:679-688

Gong P, van Leeuwen C (2004) Evolution to a small-world network with chaotic units Europh Lett 6:328-333

Gulati R, Sytch M, Tatarynowicz A (2012) The rise and fall of small worlds: Exploring the dynamics of social structure. Organiz Sc 23:449-471

Hagberg AA, Swart PA, Chult DS (2008) Exploring network structure, dynamics, and function using networkx. in G Varoquaux, T Vaught, J Millman (Eds), Proc 7th Python in Sc Conf (SciPy2008). (Pasadena, CA USA), pp. 11--15

Ikegaya Y, Sasaki T, Ishikawa D, Honma N, Tao K, Takahashi N, Minamisawa G, Ujita S, Matsuki N (2013) Interpyramid spike transmission stabilizes the sparseness of recurrent network activity. Cereb Cortex 23:293-304

Ito J, Kaneko K (2002) Spontaneous structure formation in a network of chaotic units with variable connection strengths. Ph Rev Lett 88:028701

Ito J, Nikolaev AR, van Leeuwen C (2007) Dynamics of spontaneous transitions between global brain states. Hum Br Mapp 28:904-913

Ito J, Nikolaev AR, van Leeuwen C (2005). Spatial and temporal structure of phase synchronization of spontaneous EEG alpha activity. Biol Cybern 92:54-60

Jarman N, Steur E, Trengove C, Tyukin I, van Leeuwen C (2017) Self-organisation of smallworld networks by adaptive rewiring in response to graph diffusion. Scient Rep 7:13518

Jones E, Oliphant E, Peterson P, et al. (2001-) SciPy: Open Source Scientific Tools for Python, http://www.scipy.org/ [Online; accessed 2018-07-13].

Kaneko K (1992) Overview of coupled map lattices Chaos 2:279-282

Kwok HF, Jurica P, Raffone A, van Leeuwen C (2006) Robust emergence of small-world structure in networks of spiking neurons. Cogn Neurodyn 1:39-51

Lang J, De Sterck H, Kaiser JL, Miller JC (2017) Random spatial networks: small worlds without clustering, traveling waves, and hop-and-spread disease dynamics. arXiv: 1702.01252 [q-bio.QM]

Muldoon SF, Bridgeford EW, Bassett DS (2016) Small-World Propensity and Weighted Brain Networks. Scientific Reports 6:22057

Munilla J, Ortiz A, Górriz JM, Ramírez J, (2017) Construction and Analysis of Weighted Brain Networks from SICE for the Study of Alzheimer's Disease. Frontiers in Neuroinformatics 11:19

Newman MEJ (2004) Analysis of weighted networks. Phys Rev E 70:5

Newman MEJ (2006) Modularity and community structure in networks. Proc Nat Acad Sc USA 103:8577-8582.

Papadopoulos L, Kim JZ, Kurths J, Bassett DS (2017) Development of structural correlations and synchronization from adaptive rewiring in networks of kuramoto oscillators, Chaos 27: 073115

Roxin A, Brunel N, Hansel D, Mongillo G, van Vreeswijk C (2011) On the distribution of firing rates in networks of cortical neurons J Neurosc 31:16217-16226

Rubinov M, Sporns O (2010) Complex network measures of brain connectivity: uses and interpretations, NeuroImage 52:1059-1069

Rubinov M, Sporns O, van Leeuwen C, Breakspear M (2009) Symbiotic relationship between brain structure and dynamics. BMC Neurosc 10:55 
Saramäki J, Kivela M, Onnela J-P, Kaski K, Kertesz J (2007) Generalizations of the clustering coefficient to weighted complex networks Phys Rev E 75: 027105.

Seidkhani H, Nikolaev AR, Meghanathan RN, Pezeshk H, Masoudi-Nejad A, van Leeuwen C (2017) Task modulates functional connectivity networks in free viewing behavior NeuroImage 159:289-301

Song S, Sjöström PJ, Reigl M, et al. (2005). Highly nonrandom features of synaptic connectivity in local cortical circuits. PLoS Biol 3:e68

Sporns O, Tononi G, Edelman GM (2000) Theoretical neuroanatomy: Relating anatomical and functional connectivity in graphs and cortical connection matrices $\mathrm{Cer}$ Cort 10:127-141

van den Berg D, van Leeuwen C (2004) Adaptive rewiring in chaotic networks renders smallworld connectivity with consistent clusters Europh Lett 65:459-464

van den Berg D, Gong P, Breakspear M, van Leeuwen C (2012) Fragmentation: loss of global coherence or breakdown of modularity in functional brain architecture? Front Syst Neurosc 6:20

van den Heuvel MP, Sporns O (2011) Rich-club organization of the human connectome. The Journal of Neuroscience 31:15775-15786

van den Heuvel MP, Sporns O, Collin G, Scheewe T, Mandl RCW, Cahn W, Goni J, Hulshoff Pol HE, Kahn RS (2013) Abnormal rich club organization and functional brain dynamics in schizophrenia. JAMA Psych 70:783-792

van Leeuwen C (2014) Ten years of adaptive rewiring networks in cortical connectivity modeling. Progress and perspectives. Cogn Proc 15:146-148

Varshney LR, Chen BL, Paniagua E, Hall DH, Chklovskii DB (2011) Structural properties of the caenorhabditis elegans neuronal network. PLoS CB 7:e1001066

Watts DJ, Strogatz SH (1998) Collective dynamics of 'small-world' networks. Nature 393:440-442 